\title{
DIVERSITY OF CONVERGENCE AND SPATIAL DEPENDENCE BETWEEN EUROPEAN METROS AND NON-METROS. AN APPLICATION OF THE TWO-REGIME SPATIAL AUTOREGRESSIVE PANEL MODEL
}

The aim of the paper is to analyse the difference in the speed of convergence and instability of spatial dependence between the metro and non-metro subregions. The tworegime spatial autoregressive panel data model estimated in the Bayesian approach using a Markov Chain Monte Carlo technique was applied for both purposes. The model was evaluated in the small Monte Carlo simulation. The main analysis was performed at the NUTS3 level, which allowed delimitating the metropolitan areas according to the Larger Urban Zone definition. A balanced panel consisted of data for 31 European countries for the years 2000-2010. A significant catching up process was found for both metros and nonmetros. However, our evidence confirms the lower speed of convergence for metros than for non-metros. The significant parameter for spatial interactions was found only for the nonmetros. The results indicate the crucial role of national factors for identifying inequalities in the convergence and spatial dependence.

Keywords: metro region, convergence, spatial panel data model, spatial regime, MCMC

JEL Classifications: C21, C23, O47, R11

DOI: $10.15611 /$ aoe.2017.2.10

\section{INTRODUCTION}

In the traditional model of neoclassical economics, convergence is defined as the dynamic process of achieving a long-term balance. The idea of convergence is based on the assumption of decreasing returns on capital. The process of reducing the level of economic growth inequalities and the development gap between the regions results from a declining willingness to invest in richer regions in favour of regions with lower levels of economic growth, where a rate of return on investments is higher (Geodecki, 2006, p. 76). Hence the dynamics of the growth rate in richer regions decreases,

\footnotetext{
* Department of Spacial Econometrics, University of Lodz.

${ }^{* *}$ Regional Territorial Observatory of Lodzkie Voivodeship, Spatial Planning Office of Lodzkie Voivodeship in Lodz.
} 
which in turn leads to the equalization of the development level between the regions.

According to Williamson (1965), the processes of divergence and convergence are typical for the stages of the countries development. In countries at an early stage of development there are large disparities of development level between its regions. While in countries with a higher level of development, the differences between the metropolitan areas and the peripheral regions decline with the decrease in the concentration of economic growth. Moreover, in highly developed countries the metropolitan areas may record a lower dynamics of economic growth in comparison to other regions. This phenomenon is connected with the increasing availability of agglomeration benefits (the better availability of the surrounding business institutions, communication, transport infrastructure, education and highly skilled workforce) outside the metropolitan areas (Dijkstra, 2009).

It seems that the rate of convergence for metros and non-metros will not be the same. On the one hand, economic growth will favour metros in less developed countries, while in areas with a higher level of development the non-metros will achieve larger growth. On the other hand metropolitan areas differ from non-metropolitan in terms of the existence of specific functions, the scope of spatial interactions, character, position in the network of economic relations, or even the level of education and the catch-up potential as a result.

The article refers to the issue of heterogeneity of the $\beta$-convergence process and spatial dependence in the area of Europe. The main aim of this study is to verify the difference between the metro and non-metro subregions of 31 European countries in the occurrence and speed of the conditional $\beta$ convergence for the period 2000-2010. Contrary to the previous papers we concentrate on the consequences of using the country-specific effects and spatial regimes in the evaluation of the convergence process. The additional aim of the research is to verify whether the spatial interaction parameter varies for the metros and non-metros. We proposed a two-regime spatial autoregressive panel model with spatial and time fixed effects estimated using the Bayesian MCMC method for both purposes. Moreover the evaluation of the model using a Monte Carlo simulation is added.

The rest of the paper is as follows. Section 2 is a brief literature review focused on those studies in which the $\beta$-convergence processes are explored using the NUTS3 (subregional) level. In Section 3, the two-regime spatial autoregressive panel model, the method of estimation, as well as the database were described. The results from the Monte Carlo simulation are 
discussed in Section 4, while Section 5 submits the empirical results for the $\beta$-convergence and spatial dependence evaluation. Further discussion about the heterogeneity of the $\beta$-convergence and instability of the spatial relations are in Section 6. Finally, the main conclusions follow.

\section{LITERATURE REVIEW}

The vast majority of the empirical studies were devoted to convergence, analyses the catch-up effects only at the regional (NUTS 2) level. Among the studies dealing with the issues of equalizing the development levels of subregions (NUTS 3), one can indicate, among others, the work by Pukeliene and Butkus (2012). They evaluated the $\beta$ convergence processes for the sample of 1190 EU27 territorial units in 1995-2008. The obtained results showed a small rate of convergence, which in the case of the analysis of the entire sample amounted to only $0.6 \%$ per year. What is important is that the rate of the absolute (unconditional) convergence differed in the analyses of national sub-samples and in the case of some countries demonstrated a statistically significant divergence.

Also, Paas et al. (2007) proved the existence of a small rate of unconditional $\beta$-convergence for the sample of 1214 EU25 subregions (for the period 1995-2002). They also noted that the speed of catching up with regions characterized by a high level of GDP by the economically backward regions was higher in the EU15 countries in comparison to the new member states. The authors took into account the effect of affiliation of a region to a given country (as the country dummies) which had a significant impact on the obtained results and drew conclusions concerning the regional convergence process. The rate of the conditional $\beta$-convergence was lower than the rate of the unconditional convergence, and in the case of selected countries one could even observe a divergence of the developmental processes, proving the existence of the polarization of areas and their division into centres and peripheries.

The mentioned studies suggest that the convergence processes observed at regional level are rather the result of the equalization of the development levels between the countries, and do not apply directly to the elimination of regional differences. The differences seem to be deepening, which is equivalent to the increase of heterogeneity inside the country. The existence of divergence tendencies within the country is also confirmed by studies which analysed a sample of territorial units for one country, e.g. those relating to Polish provinces (Dańska-Borsiak, 2011), regions of Portugal 
and Spain (Viegas, Antunes 2013) and regions of Russia (Akhmedjonov et al., 2012).

The studies carried out for NUTS3 spatial aggregation level have rarely taken into account the diversity of convergence processes within the subgroups of territorial units. Among the studies one should mention the work by Frenken and Hoekman (2006), who from the sample of 1088 EU25 subregions selected areas which could be defined as network cities. The results obtained by them showed a significantly higher rate of growth of network cities, compared to the remaining areas. Moreover, they noted that conclusions regarding the convergence or divergence depended on the level of spatial aggregation of data and differed between the EU15 countries (regional convergence) and the new members of the $\mathrm{EU}$ (divergence).

Chapman et al. (2012) also noted that the spatial heterogeneity of the development processes, is leading to the creation of the so-called clubs (club convergence). As far as the capital cities and urban regions are concerned, the researchers confirmed that they stood out from the periphery in terms of the growth level, but they were not given much attention. They were only taken into account as additional binary explanatory variables in spatial models.

A few examples of analyses emphasizing the division of regions into metropolitan and non-metropolitan ones for Europe (to be precise, for the EU) are complemented by studies on the U.S.. In particular, Hammond (2006) proved the existence of divergence for the non-metropolitan areas in 1969-2001, while the metropolitan areas recorded a diversity in convergence processes, which depended on their geographical location and size. The fastest speed of convergence could be observed in the case of medium-sized metropolis, while the strongest divergence tendencies were characteristic for non-metropolitan areas within which large urban centres and small towns were located.

In accordance with the studies presented in the literature, both interregional interactions (spatial effects) and location in a given country (spatial heterogeneity) may play an important role in the analysis of regional convergence processes. In particular, these factors affect the inference of the existence of economic convergence. In addition, one can note that the convergence processes may differ in the case of a division between development centres and peripheral areas. 


\section{METHOD AND DATA}

\subsection{Two-regime spatial autoregressive panel model with fixed effects}

The traditional fixed and random effects panel models are able to capture the unobserved heterogeneity of the regional economies. Such individual effects are most often identified as the initial level of technology in the convergence analysis (Badinger et al., 2002). The common practice is also adding time effects which are responsible for the common time tendency of regional growth (business cycle). As the lack of information about the time volatility and individual characteristics of spatial unit leads to increasing the value of residuals and their correlation with the explanatory variables, and also to inconsistency and biasness of the estimates (Caselli et al., 1996), the panel data model seems to be the obvious starting point in the convergence analysis.

In recent years spatial (and spatial dynamic) panel models have started to play a major role (see Yu and Lee, 2012; Bouayad-Agha and Védrine, 2010, among others). One of their advantages is the possibility of assuming the existence of relationships between economies. This is because of the fact that necessary conditions for the economic convergence are, for example, mobility of production factors, trade ties, and diffusion of knowledge and technology. As the direct measurement of the above determinants is difficult or even impossible, such interactions are included in an indirect manner using a priori specified spatial weight matrix.

Following the highlighted problems, we proposed an alternative way of the convergence analysis which is based on the assumption about the heterogeneity of the convergence process. This is because of the club convergence which was found in the previous studies (e.g. Fischer and Stirböck, 2006; Jabłoński, 2012; Pietrzak, 2012). Such club convergence means the difference between groups of regions (or countries) in the speed of the convergence or even in the convergence versus divergence existence. In our model all spatial units which belong to the same homogenous club (spatial regime) are going to the same long-term balance which differs between clubs. In this study we classified all subregions into two groups, metro and non-metro, which we expected to find as the two clubs with a different speed of convergence process.

Moreover, we assumed the heterogeneity of the spatial dependence among those groups and we supposed that spatial spillover differs between metro and non-metro areas because the impact of neighbours on metro and 
non-metro regions is not the same. That results in the instability of the spatial effect parameter, or in other words the spatial heterogeneity in the form of spatial regimes.

Due to the above, the diversity between metro and non-metro areas was incorporated as two spatial regimes, existing in both the convergence and spatial interaction parameter. We used the Barro and Sala-i-Martin (1992) approach to specify the two-regime spatial autoregressive panel data model with both spatial and time fixed effects. This can be expressed as:

$$
\begin{gathered}
\ln \left(\frac{y_{i j, t}}{y_{i j, t-1}}\right)=\beta_{1} d_{i j} \ln \left(y_{i j, t-1}\right)+\beta_{2}\left(1-d_{i j}\right) \ln \left(y_{i j, t-1}\right)+ \\
\mu_{i}+\rho_{1} d_{i j} \sum_{i=1}^{N} w_{i j} \mu_{i+1 j}+\rho_{2}\left(1-d_{i j}\right) \sum_{i=1}^{N} w_{i j} \mu_{i+1 j}+\tau_{t}+v_{j}+\varepsilon_{i j, t}, \\
\varepsilon_{i j, t} \sim N\left(0, \sigma_{\varepsilon}^{2}\right), \quad \mu_{i j} \sim N\left(0, \mathbf{I}_{N} \sigma_{\mu}^{2}\right),
\end{gathered}
$$

where: $i j$ - cross-sectional dimension (subregions and countries), with $i=1, \ldots, N$ subregions and $j=1, \ldots, J$ countries, $t$ - time dimension, with $t=1, \ldots, T, y_{i j, t}$ GDP per inhabitant, $\tau_{t}$ - time-period fixed effect, $v_{j}-$ country-specific fixed effect, $\mu_{i}$ - random subregional effect, $d_{i j}$ - binary variable for the spatial regimes which takes the value of 1 if subregion $i$ was classified as metropolitan area, and 0 otherwise (the same in each $t$ ), $\beta_{1}, \beta_{2}$ - estimated parameters for metro and non-metro subregions which inform about the convergence $(\beta<0)$ or divergence $(\beta>0), \rho_{1}, \rho_{2}$ - estimated spatial effect parameters for the metropolitan and non-metropolitan regime, $\sum_{i=1}^{N} w_{i j}-$ elements of the row-standardized spatial weight matrix $\mathbf{W}$.

The elements $w_{i j}$ were calculated using the inverse-square function of a geographical distance $\left(d_{i j}\right)$ between the centroids of subregions:

$$
w_{i j}=\left\{\begin{array}{cl}
1 / d_{i j}^{2} & \text { if } d_{i j} \leq h ; \\
0 & \text { if } d_{i j}>h .
\end{array}\right.
$$

The critical cut-off distance $h=200 \mathrm{~km}$ was set to achieve a more semilocal structure of the spatial relations. We used the results from the variogram to obtain the cut-off distance. As a result we achieved the spatial structure with the mean number of neighbours per subregion equal to 63 (4.6\% of the whole sample). The semi-local structure means that for most of 
the subregions (70\%), the neighbours are not only from the same country but also from neighbouring foreign countries.

Based on the estimated $\beta_{1}, \beta_{2}$ parameters (Equation 1), the rate of the convergence (annual rate of achieving the long-term balance) was calculated for both regimes. Additionally, the half-time rate (Barro, Sala-i-Martin, 1995) was used to compare the period during which the distance between the metro (non-metro) economies and the common long-term balance level will be reduced by a half. The difference between $\beta_{1}$ and $\beta_{2}$ was calculated to check if the metro and non-metro subregions vary in the convergence rate. We compared them using the credible interval (CI) of the $\beta$ parameters for which the coverage was checked. By analogy, we tested the difference of the spatial interaction parameters for the metro and non-metro subregions.

In Equations 3 and 4 the unexplained changes in the level of dependent variable due to the specificity of the following years was captured by time fixed effects. Additionally, subregional units interactions in the geographical space might more or less depend on the metropolitan or non-metropolitan status of the unit. In contrast to the multiparametric spatiotemporal autoregressive model (Hays et al., 2009), for each subregion the same structure of spatial dependence was assumed. Hence the source of the metropolitan and non-metropolitan area spatial effect difference is not the way they interact in the space but sensitivity for the economic development in the neighbourhood. Our approach is different from the traditional spatially switching regression (Anselin, 1988, pp. 132-133), where $\sigma_{\varepsilon}^{2}$ varies between subgroups. We assumed like Elhorst and Fréret (2009) that $\sigma_{\varepsilon}^{2}$ does not differ between the regimes. Also $\sigma_{\mu}^{2}$ is the same in the metro and non-metro subgroups.

\subsection{Bayesian Markov Chain Monte Carlo estimation}

As opposed to Elhorst and Fréret (2009) who estimated a two-regime Durbin model with fixed effects via maximum likelihood (MLE), the Bayesian Markov Chain Monte Carlo (MCMC) method was applied to estimate Equation 1, as more flexible and preferred for the complex model (see LeSage and Pace, 2009). Typically for the Bayesian perspective we assumed that the joint probability distribution of the all parameters $\boldsymbol{\theta}^{*}$ and observed data $\mathbf{D}^{*}$ consist of two parts: the prior distributions $P\left(\boldsymbol{\theta}^{*}\right)$ and a likelihood $P\left(\mathbf{D}^{*} \mid \boldsymbol{\theta}^{*}\right)$, which can be written as:

$$
P\left(\mathbf{D}^{*}, \boldsymbol{\theta}^{*}\right)=P\left(\mathbf{D}^{*} \mid \boldsymbol{\theta}^{*}\right) P\left(\boldsymbol{\theta}^{*}\right),
$$


where: $\boldsymbol{\theta}^{*}=\left\{\boldsymbol{\beta}, \boldsymbol{\rho}, \sigma_{\varepsilon}{ }^{2}, \sigma_{\mu}{ }^{2}\right\}, \mathbf{D}^{*}=\{\mathbf{y}, \mathbf{X}, \mathbf{W}\}$. Then, we specified the prior distribution and derived the conditional posterior distribution for each parameters.

We used Harris and Dong's (2014) detailed description of the Bayesian implementation for the hierarchical spatial autoregressive model (HSAR) because we found it is similar to our model (Equation 1). The spatiotemporal structure might be considered as a two-level in the multilevel framework. Hence, it is possible to treat Equation 1 as the multiple spatial weights extension of the HSAR model. The main difference between both is the lack of the time (the synonym of individual) level spatial interactions in Equation 1 and the separation of the spatial effect for the two regimes (metropolitan and non-metro) at the second (subregional) level. Despite this, the specification of the prior and posterior distributions of parameters in the two-regime spatial autoregressive panel model are like in the HSAR model.

Accordingly, we set up the prior distribution of fixed and random effect parameters as:

$$
\begin{aligned}
& P(\boldsymbol{\beta}) \sim N\left(\mathbf{M}_{0}, \mathbf{T}_{0}\right), \\
& P(\boldsymbol{\rho}) \sim U\left(1 / v_{\rho \min }, 1\right), \\
& P\left(\sigma_{\varepsilon}^{2}\right) \sim I G\left(c_{0}, d_{0}\right), \\
& P\left(\sigma_{\mu}^{2}\right) \sim I G\left(a_{0}, b_{0}\right),
\end{aligned}
$$

where: $\boldsymbol{\beta}=\left[\beta_{1}, \beta_{2}, \tau_{1}, \ldots, \tau_{T-1}, v_{1}, \ldots, v_{J-1}\right]^{\prime}-K \times 1$ vector of fixed effect parameters, $\mathbf{M}_{0}-K \times 1$ vector of means, $\mathbf{T}_{0}-K \times K$ variance-covariance matrix, $v_{\min }$ - the minimum eigenvalue of the weight matrix $\mathbf{W}, K$ - total number of fixed effect parameters in the model, $\boldsymbol{\rho}=\left[\rho_{1}, \rho_{2}\right]^{\prime}-2 \times 1$ vector of spatial effect parameter for both spatial regimes, $I G$ - the inverse gamma distribution with the shape parameter $a_{0}$ or $c_{0}$ and scale parameter $b_{0}$ or $d_{0}, N$ - the normal distribution, $U$ - the uniform distribution. The fairly noninformative priors with the distributions as in Equation 4 were used to draw the initial values in the MCMC.

To derive the conditional posterior distribution from fixed and random parameters, full posterior distribution for the estimated parameters was taken. Consistent with Equation 3 it can be expressed as:

$$
\begin{aligned}
& P\left(\boldsymbol{\rho}, \boldsymbol{\beta}, \boldsymbol{\theta}, \sigma_{\mu}^{2}, \sigma_{\varepsilon}^{2} \mid \mathbf{y}\right) \propto L\left(\mathbf{y} \mid \boldsymbol{\rho}, \boldsymbol{\beta}, \boldsymbol{\theta}, \sigma_{\mu}^{2}, \sigma_{\varepsilon}^{2}\right) \times \\
& P(\boldsymbol{\beta}) \times P(\boldsymbol{\rho}) \times P\left(\boldsymbol{\theta} \mid \boldsymbol{\rho}, \sigma_{\mu}^{2}\right) \times P\left(\sigma_{\mu}^{2}\right) \times P\left(\sigma_{\varepsilon}^{2}\right)
\end{aligned}
$$


with the likelihood function that indicates $P\left(\mathbf{D} \mid \boldsymbol{\theta}^{*}\right)$ :

$$
\begin{gathered}
L\left(\mathbf{y} \mid \boldsymbol{\rho}, \boldsymbol{\beta}, \boldsymbol{\theta}, \sigma_{\mu}^{2}, \sigma_{\varepsilon}^{2}\right)=\left(2 \pi \sigma_{\varepsilon}^{2}\right)^{-N T / 2} \exp \left\{-\frac{1}{2 \sigma_{\varepsilon}^{2}}(\mathbf{y}-\boldsymbol{\beta} \mathbf{X}-\boldsymbol{\Delta} \boldsymbol{\theta})^{\prime}(\mathbf{y}-\boldsymbol{\beta} \mathbf{X}-\boldsymbol{\Delta} \boldsymbol{\theta})\right\}, \\
\boldsymbol{\theta}=\left(\mathbf{I}_{N}-\rho_{1} \mathbf{D W}\right)^{-1} \boldsymbol{\mu}+\left(\mathbf{I}_{N}-\rho_{2}(\mathbf{1}-\mathbf{D}) \mathbf{W}\right)^{-1} \boldsymbol{\mu},
\end{gathered}
$$

where: $\mathbf{y}=\left[y_{i j, t}\right]^{\prime}-N T \times 1$ vector of dependent variables, $\mathbf{1}-N \times 1$ vector of one's, $\mathbf{I}_{N}-$ identity matrix, $\boldsymbol{\mu}=\left[\mu_{i}\right]^{\prime}-N \times 1$ vector of spatial random effects, $\boldsymbol{\Delta}-N T \times N$ block-diagonal design matrix, $\mathbf{D}=\left[d_{i j, 1}, \ldots, d_{i j, T}\right]^{\prime}-N T \times 1$ vector for identifying the spatial regimes.

Due to the above, the posterior distribution of parameters were as follows (compare with Harris and Dong, 2014):

$$
P\left(\boldsymbol{\beta} \mid \mathbf{y}, \boldsymbol{\rho}, \boldsymbol{\theta}, \sigma_{\varepsilon}^{2}, \sigma_{\mu}^{2}\right) \sim N\left(\mathbf{M}_{\beta}, \boldsymbol{\Sigma}_{\beta}\right),
$$

where:

$$
\begin{aligned}
& \boldsymbol{\Sigma}_{\beta}=\left[\left(\sigma_{\varepsilon}^{2}\right)^{-1} \mathbf{X}^{\prime} \mathbf{X}+\mathbf{T}_{0}^{-1}\right]^{-1}, \\
& \mathbf{M}_{\beta}=\boldsymbol{\Sigma}_{\beta}\left[\left(\sigma_{\varepsilon}^{2}\right)^{-1} \mathbf{X}^{\prime}(\mathbf{y}-\Delta \boldsymbol{\theta})+\mathbf{T}_{0}^{-1} \mathbf{M}_{0}\right] \\
& P\left(\boldsymbol{\theta} \mid \mathbf{y}, \boldsymbol{\rho}, \boldsymbol{\beta}, \sigma_{\varepsilon}^{2}, \sigma_{\mu}^{2}\right) \sim N\left(\mathbf{M}_{\boldsymbol{\theta}}, \Sigma_{\boldsymbol{\theta}}\right),
\end{aligned}
$$

where:

$$
\begin{gathered}
\Sigma_{\theta}=\left[\left(\sigma_{\varepsilon}^{2}\right)^{-1} \Delta^{\prime} \Delta+\left(\sigma_{\mu}^{2}\right)^{-1} \mathbf{B}^{\prime} \mathbf{B}\right]^{-1}, \\
\mathrm{M}_{\boldsymbol{\theta}}=\Sigma_{\theta}\left[\left(\sigma_{\varepsilon}^{2}\right)^{-1} \Delta^{\prime}(\mathbf{y}-\mathbf{X} \boldsymbol{\beta})\right] \\
P\left(\sigma_{\mu}^{2} \mid \mathbf{y}, \boldsymbol{\rho}, \boldsymbol{\beta}, \boldsymbol{\theta}, \sigma_{\varepsilon}^{2}\right) \sim I V\left(a_{\mu}, b_{\mu}\right),
\end{gathered}
$$

where:

$$
\begin{gathered}
a_{\mu}=J / 2+a_{0}, \\
b_{\mu}=\boldsymbol{\theta}^{\prime} \mathbf{B}^{\prime} \mathbf{B} \boldsymbol{\theta} / 2+b_{0} . \\
P\left(\sigma_{\varepsilon}^{2} \mid \mathbf{y}, \boldsymbol{\rho}, \boldsymbol{\beta}, \boldsymbol{\theta}, \sigma_{\mu}^{2}\right) \sim I V\left(c_{\varepsilon}, d_{\varepsilon}\right),
\end{gathered}
$$

where:

$$
\begin{aligned}
& c_{\varepsilon}=N / 2+c_{0}, \\
& d_{\varepsilon}=0,5 \times(\mathbf{y}-\mathbf{X} \boldsymbol{\beta}-\Delta \boldsymbol{\theta})^{\prime}(\mathbf{y}-\mathbf{X} \boldsymbol{\beta}-\boldsymbol{\Delta} \boldsymbol{\theta})+d_{0} .
\end{aligned}
$$

where: $\mathbf{B}=\mathbf{I}_{N}-\rho_{1} \mathbf{D W}-\rho_{2}(\mathbf{I}-\mathbf{D}) \mathbf{W}$. 
Gibbs sampling was employed to draw the samples for parameters. This means that in each iteration the new values of parameters were drawn from their conditional distribution using the current values of all other parameters (Nielsen, 2006, p. 49). The lack of the standard recognizable density distributions for $\rho_{1}$ and $\rho_{2}$ resulted in that we decided to apply the inverse sampling method to update both spatial interaction parameters. In each iteration the numerical integration of $\log f\left(\rho_{1}\right)$ over $\left(1 / v_{\rho 1 \text { min }}, 1\right)$ and $\log f\left(\rho_{2}\right)$ over $\left(1 / v_{\rho 2 \min }, 1\right)$ was computed. After that the cumulative distribution of $\rho_{1}$ and $\rho_{2}$ were calculated and the inverse sampling approach was employed to draw values of parameters. The $\log f\left(\rho_{1}\right)$ and $\log f\left(\rho_{2}\right)$ are as follows:

$$
\begin{aligned}
& \log f\left(\rho_{1}\right)=\log \left|\mathbf{I}_{N}-\rho_{1} \mathbf{D W}\right|+\boldsymbol{\theta}_{1}{ }^{\prime} \mathbf{B}_{1}{ }^{\prime} \mathbf{B}_{1} \boldsymbol{\theta}_{1} / 2 \sigma_{\mu}^{2}, \\
& \log f\left(\rho_{2}\right)=\log \left|\mathbf{I}_{N}-\rho_{2}(\mathbf{l}-\mathbf{D}) \mathbf{W}\right|+\boldsymbol{\theta}_{2}{ }^{\prime} \mathbf{B}_{2}{ }^{\prime} \mathbf{B}_{2} \boldsymbol{\theta}_{2} / 2 \sigma_{\mu}^{2},
\end{aligned}
$$

where: $\mathbf{B}_{1}=\mathbf{I}_{N}-\rho_{1} \mathbf{D W}, \mathbf{B}_{2}=\mathbf{I}_{N}-\rho_{2}(\mathbf{I}-\mathbf{D}) \mathbf{W}, \boldsymbol{\theta}_{1}=\left(\mathbf{I}_{N}-\rho_{1} \mathbf{D W}\right)^{-1} \boldsymbol{\mu}, \boldsymbol{\theta}_{2}=$ $\left(\mathbf{I}_{N}-\rho_{2}(\mathbf{I}-\mathbf{D}) \mathbf{W}\right)^{-1} \boldsymbol{\mu}$.

The MCMC sampler was coded using the R language. The convergence of the MCMC sampler was diagnosed using the CODA package in R Cran (Plummer et al., 2006). The inferences were based on one MCMC chain, each consisting of 10,000 iterations with a burn-in period of 5,000.

\subsection{Data}

We used data for the years 2000-2010 at the NUTS3 level for 28 European Union countries, and also Switzerland, Norway and Macedonia. These data came from Eurostat, the BADAC Database of Swiss Cantons and Towns (for Switzerland), the Republic of Macedonia State Statistical Office (price index for Macedonia) and the OECD database (Norway for the period 2000-2007). Changes in the NUTS classification (e.g. in the case of Germany, Italy, the Netherlands and Croatia), and in particular changes in the boundaries, the merger and separation of new subregions were taken into account by the recalculation of the variable values according to the NUTS classification from 2010. The only one exception was the Province of Barletta-Andria-Trani (Italy), which was omitted in the analysis due to the fact that it was sectioned off as a new territorial unit in 2008, which prevented the calculation of subregional gross domestic product per inhabitant for the period 2000-2008. 
The economic growth was expressed as the level of subregional gross domestic product (GDP) per inhabitant in PPS million, in fixed prices from the year 2000. In the calculations, the mid-year number of inhabitants was used as a proxy of subregional population and the price index as a deflator. Figure 1 and 2 present the basic descriptive statistics and values of GDP per inhabitant.

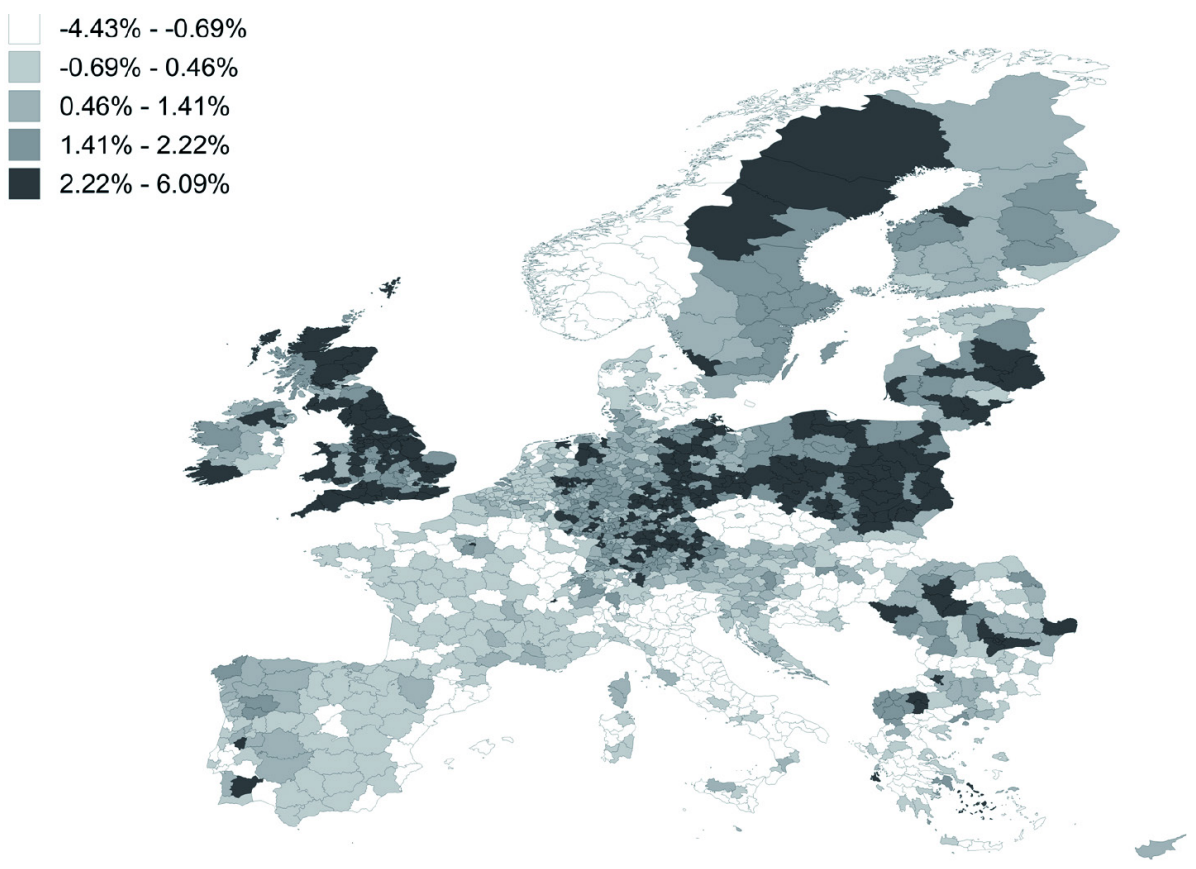

Figure 1. NUTS3 gross domestic product (average annual growth rate in \%).

Source: author's calculations based on data from Eurostat.

The division of the NUTS3 units into metropolitan and non-metropolitan subregions was based on a delimitation consistent with LUZ (Urban Audit's Larger Urban Zones), prepared under the Urban Audit Programme implemented by Eurostat. According to the method of classification of the NUTS3 units accepted by Eurostat, the status of metropolitan subregions was granted to those where at least $50 \%$ of the population live in the LUZ metropolitan areas. The latter are defined as agglomerations of at least 250 000 inhabitants (cf. Dijkstra, 2009). That gives 258 metros in the EU27, classified into three types: capital metros (the equivalent of the national 


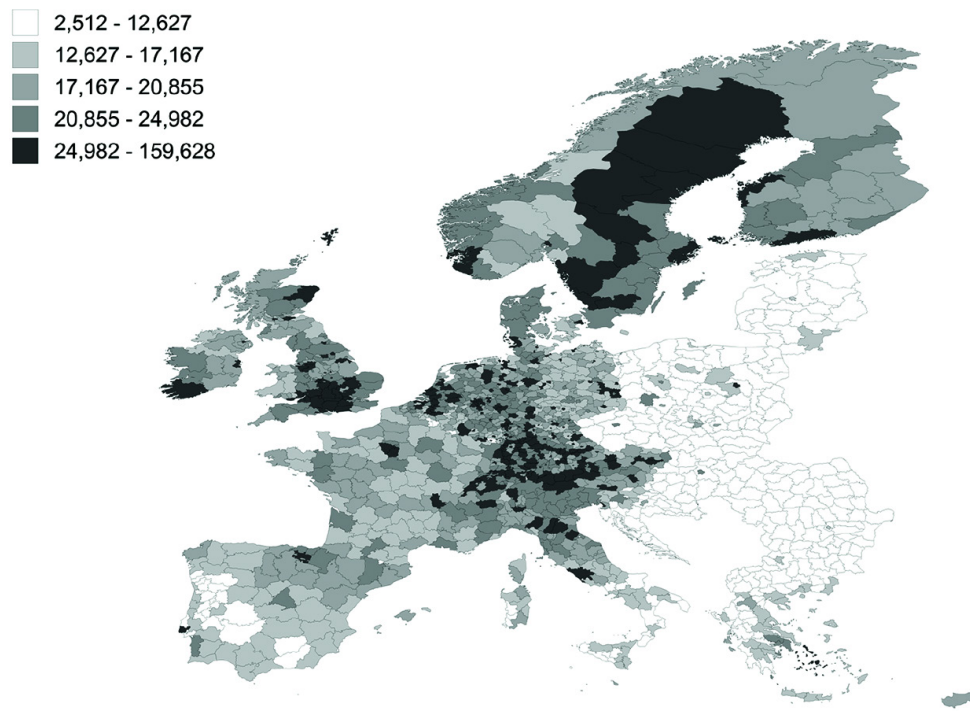

Figure 2. NUTS3 real gross domestic product in 2010 (PPS per inhabitant)

Source: author's calculations based on data from Eurostat.

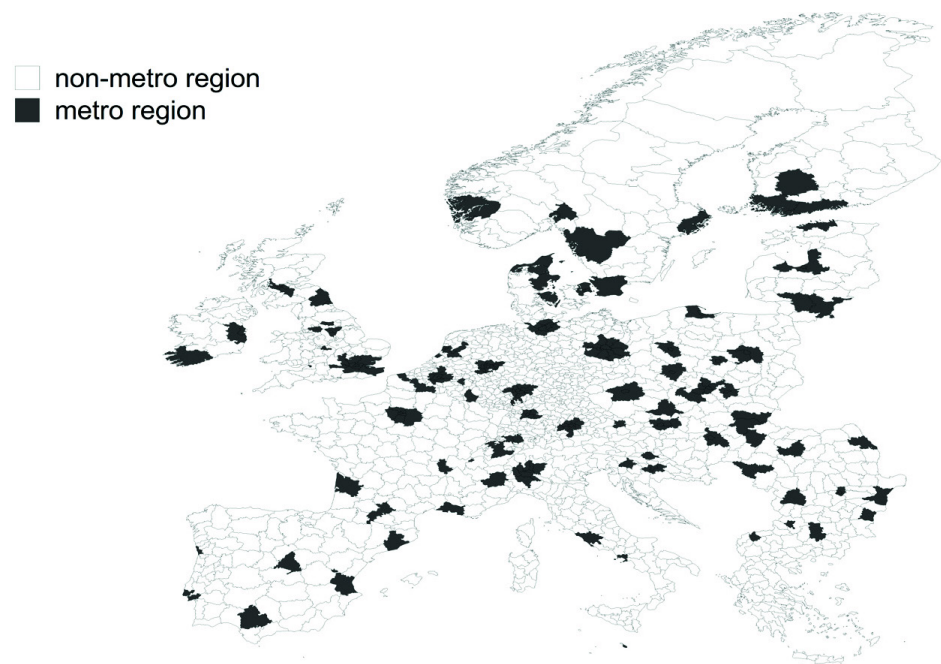

Figure 3. Metro subregions delimitation according to LUZ with the smaller metros treated as non-metros

Source: author's elaboration based on data from Eurostat. 
capitals), second tier metros (the group of the largest cities in a country, except the capital) and smaller metros. In the two first classes the most important cities for the particular country are grouped. As the fixed population threshold could not be used to separate the second tier metros for all the countries, the category of smaller metros was set up (Eurostat, 2013, p. 219). In our study, smaller metros were treated as non-metros (nonmetropolitan subregions), while the capital and the second tier units were classified to the common group called metros (metropolitan subregions). Figure 3 presents the delimitation of the metro and non-metro subregions based on the NUTS3 units.

The database (balanced panel) consists of $N T=14,993$ observations coming from $N=1,363$ NUTS3 units called subregions. According to the LUZ classification, 238 NUTS3 units were recognized as metropolitan subregions. Due to the fact that the selected metropolitan subregions consist of more than one NUTS3 territorial unit (e.g. the Zürich metropolitan area consists of Aargau and Zürich), the total number of different metros amounted to 92 .

\section{THE EVALUATION OF THE TWO-REGIME SPATIAL AUTOREGRESSIVE PANEL MODEL IN THE MONTE CARLO SIMULATION}

We started from the evaluation of our model using the Monte Carlo simulation to check for the potential biasness of the estimated parameters. Two simulation experiments were conducted with the same target value of parameters and similar number of iterations $(R=80$ and 100) but different in the data generating processes. In the first experiment (model 1) we assumed there is no spatial and time fixed effects, while in the second one (model 2) we extended the data generating process using those effects. In both experiments we took the values of independent variables from our database and the spatial weight matrix as in Equation 2. The random effects $\mu_{i}$ and error term $\varepsilon_{i j, t}$ were drawn from the normal distribution with zero mean and the variance equal one. To evaluate the models' results, the relative bias and coverage of the $95 \%$ credible interval were calculated for each estimated parameter.

The simulation results are provided in Table 1 . In both experiments all the parameters were found unbiased. The relative biases for all the parameters are less than those commonly used in the simulation studies threshold value equal to 5\% (see Hoogland and Boomsma, 1998). In model 1 
the relative biases for the estimates are lower than $1 \%$, except the estimates for the spatial interaction parameters $(1.6 \%$ and $1.8 \%)$. The coverage rates of the $95 \%$ credible interval $(\mathrm{CI})$ are also satisfactory. Both the fixed and random parameter estimates are characterized by the higher than $90 \%$ coverage rate of the $95 \% \mathrm{CI}$. Such results suggest that the credible intervals are unbiased in model 1.

Table 1

The evaluation of the two-regime spatial random effect model using Monte Carlo simulation

\begin{tabular}{|c|c|c|c|}
\hline Parameter & Target value & $\begin{array}{l}\text { Relative bias } \\
\quad \text { (in \%) }\end{array}$ & $\begin{array}{l}\text { Coverage rate } \\
\text { (in \%) }\end{array}$ \\
\hline \multicolumn{4}{|c|}{ Model $1(R=80)$} \\
\hline$\beta_{1}$ & -0.020 & -0.007 & 92.500 \\
\hline$\beta_{2}$ & -0.030 & 0.002 & 92.500 \\
\hline$\rho_{1}$ & 0.400 & 1.568 & 96.250 \\
\hline$\rho_{2}$ & 0.200 & 1.771 & 95.000 \\
\hline Spatial FE & No & & \\
\hline Time FE & No & & \\
\hline$\overline{\sigma_{\mu}{ }^{2}}$ & 0.001 & 0.001 & 95.000 \\
\hline$\sigma_{\varepsilon}{ }^{2}$ & 0.003 & 0.001 & 91.250 \\
\hline \multicolumn{4}{|c|}{ Model $2(R=100)$} \\
\hline$\beta_{1}$ & -0.020 & 0.191 & 85.000 \\
\hline$\beta_{2}$ & -0.030 & 0.301 & 83.000 \\
\hline$\rho_{1}$ & 0.400 & -2.392 & 82.000 \\
\hline$\rho_{2}$ & 0.200 & -0.201 & 78.000 \\
\hline \multicolumn{4}{|l|}{ Two-regime ${ }^{*}$ : } \\
\hline Spatial FE & 1.000 & 0.209 & 95.814 \\
\hline Time FE & 1.000 & -0.173 & 94.944 \\
\hline$\sigma_{\mu}{ }^{2}$ & 0.001 & -0.009 & 82.000 \\
\hline$\sigma_{\varepsilon}^{2}$ & 0.003 & -0.030 & 80.000 \\
\hline
\end{tabular}

* The relative bias and coverage rate calculated as a mean for all spatial (time) effects.

Source: author's calculations.

In the second experiment (model 2), we added both spatial and time fixed effects with metro and non-metro regimes. Again, we found the relative bias for the estimated parameters lower than 5\%. For each estimated parameter the relative bias is less than $1 \%$. The only exception is the estimated parameter for the spatial interaction $\left(\rho_{1}\right)$ which is underestimated by more than $2 \%$. Although the accuracy of the estimates was proved for model 2, the relative biases are higher than in model 1 . Also the coverage rate of the $95 \%$ 
$\mathrm{CI}$ is much lower for model 2 estimates than in the previous one. This might be the result of the correlation with the spatial and time fixed effects. Despite this, as long as the estimates are unbiased, we can use our model to test the heterogeneity of the convergence and instability of the spatial interaction parameter.

\section{EMPIRICAL RESULTS}

The empirical results were presented in three subsections. The first two with the models which were obtained by adding the restrictions on the tworegime spatial autoregressive panel model parameters (random effect models and spatial random effect models) and the third with the two-regime spatial autoregressive panel model.

\subsection{Random effect models}

Firstly, we estimated the random effect models using the Bayesian MCMC method. We achieved them as the restricted two-regime spatial autoregressive panel model in which there is no spatial dependence $\rho_{1}=\rho_{2}=0$. Two versions of the random effect model were estimates: with one-regime spatial and time fixed effects (1) and with two-regime fixed effects (2). Despite this, in both models we separated the $\beta$-convergence parameter into metro and non-metro subregions. Table 2 contains the results corresponding to these subsections.

The estimated parameters $\beta 1$ and $\beta 2$ are lower than zero and significant in both models. This means we observed the conditional convergence among metro and non-metro subregions in 2000-2010. Crucial to verify our hypothesis about the heterogeneity of the convergence process is the difference between the parameters $\beta 1$ (for the metros) and $\beta 2$ (for the nonmetros). Such a difference was found as significant only for the model with two-regime spatial and time fixed effects (2). When we assume that all subregions are similar in the time fluctuation and the country-specific effects (1), the difference between the conditional $\beta$-convergence is insignificant.

The estimated variance of random effects was found as significant in the both random effect models. Its value is the same for the model with oneregime and two-regime fixed effects. The variability of the subregional GDP per inhabitant growth is only $3.2 \%$ of the total variance. Despite this, random effects let us take into account the individual characteristics of subregions and allow us to avoid the misspecification connected with the omitted 
Table 2

Estimation results for the conditional $\beta$-convergence and spatial effects using random effect and spatial random effect models

\begin{tabular}{l|c|c|c|c|c}
\hline Parameter & $\begin{array}{c}\text { Random } \\
\text { effect } \\
\text { model (1) }\end{array}$ & $\begin{array}{c}\text { Random } \\
\text { effect } \\
\text { model (2) }\end{array}$ & $\begin{array}{c}\text { Spatial } \\
\text { random effect } \\
\text { model } \\
\mathbf{( 1 )}\end{array}$ & $\begin{array}{c}\text { Spatial random } \\
\text { effect model } \\
\mathbf{( 2 )}\end{array}$ & $\begin{array}{c}\text { Spatial } \\
\text { random effect } \\
\text { model } \\
\mathbf{( 3 )}\end{array}$ \\
\hline$\beta_{1}$ & -0.022 & -0.016 & -0.022 & -0.017 & -0.019 \\
$(0.002)$ & $(0.004)$ & $(0.004)$ & $(0.004)$ & $(0.002)$ \\
\hline$\beta_{2}$ & -0.024 & -0.030 & -0.024 & -0.030 & -0.021 \\
& $(0.002)$ & $(0.002)$ & $(0.003)$ & $(0.003)$ & $(0.002)$ \\
\hline$\rho$ & & & 0.234 & 0.205 & 0.870 \\
\hline Spatial FE & Yes (1) & Yes (2) & Yes (1) & Yes (2) & No \\
\hline Time FE & Yes (1) & Yes (2) & Yes (1) & Yes (2) & Yes (2) \\
\hline$\sigma_{\mu}^{2}$ & 0.0001 & 0.0001 & 0.0001 & 0.0001 & 0.0001 \\
\hline$\sigma_{\varepsilon}^{2}$ & $(0.000)$ & $(0.000)$ & $(0.000)$ & $(0.000)$ & $(0.000)$ \\
\hline Moran's $I$ & 0.003 & 0.003 & 0.003 & 0.003 & 0.003 \\
\hline$\mu_{i}$ & $(0.000)$ & $(0.000)$ & $(0.000)$ & $(0.000)$ & $(0.000)$ \\
\hline Metros: & & & & & \\
\hline conv. speed & $2.225 \%$ & $1.613 \%$ & $2.225 \%$ & $1.715 \%$ & $1.918 \%$ \\
\hline half-time & 31.159 & 42.974 & 31.159 & 40.426 & 36.134 \\
\hline Non- & & & & & \\
metros: & & & & & \\
\hline conv. speed & $2.429 \%$ & $3.046 \%$ & $2.429 \%$ & $3.046 \%$ & $2.122 \%$ \\
\hline half-time & 28.533 & 22.757 & 28.533 & 22.757 & 32.659 \\
\hline
\end{tabular}

Posterior mean, posterior standard deviation in parentheses. (1) one-regime effects, (2) tworegime effects

Source: author's calculations.

variables and heterogeneity. Hence, even if the proportion of the total variance being the result of the difference between subregions is small but significant, we are not allowed to ignore it.

Finally, we tested estimated random effects for the presence of spatial dependence using Moran's I test. Significant spatial autocorrelation was found in both models which proves that the random effect models suffer from a misspecification due to omitted spatial dependence. As in the traditional random effect model, the independency of the random effects is assumed, we are obliged to use an alternative specification. 


\subsection{Spatial random effect models}

Next, we estimated the spatial random effect models with one-regime (1) and two-regime fixed effects (2). The spatial dependence of the random effects was incorporated as the spatial lag term. In both models, spatial interaction parameter $\rho$ was found as insignificant because zero lies in the $95 \%$ CI for the spatial parameter (see Bolstad, 2011). This might be because of the wrong way of incorporating the spatial dependence - not as the spatial lag of the dependent variable but as the spatially lagged error term. Another explanation is that spatial dependence is not observed for all subregions but only for a subsample (e.g. metros). Hence, when most of the spatial units in the sample are non-metros, the estimated spatial parameter would equal zero. This strategy of the interpretation was tested in the next subsection by diversifying the $\rho$ parameter. Finally, we might have obtained the insignificant parameter $\rho$ because of the correlation between spatial fixed effects and the spatially lagged term. Let us now consider the last explanation.

By assuming the semi-local form of the spatial relationships we obtained the spatial structure where the neighbours are only or mostly from the same country. For $30 \%$ of all spatial units in the $\mathbf{W}$ matrix the subregional GDP per inhabitant depends on the weighted average of the GDP per inhabitant for a group of units localized in the same country. This might be the source of similarities between the spatially lagged term and the spatial fixed effects. To check this, we estimated the spatial random effect model without the spatial fixed effects (3). As we expected, when the specific country effects were excluded, the estimated spatial interaction parameter was higher (almost 0.9 ) and significant. This proves the omitted country variability affects the spatial interaction parameter and results in its overestimation. Although our suspicion regarding the connections between the subregional interactions (captured by the $\rho$ parameter) and the cross-country differences (captured by the spatial FE) seems to be right, it is still hard to conclude whether the country-specific effects ( models 1 and 2) could misleadingly capture the effect of the spatial interactions.

Surprisingly, the estimated variance of the random effects does not change when we allow for the spatial dependence in model 3. Such a result is uncommon as the overestimation of the random effect variance due to ignoring spatial autocorrelation was proved in the previous studies. It additionally shows the spatial fixed effect parameters are more susceptible for the omitted spatial dependence than the random effect variance.

As is known from the literature (see Arbia et al., 2005), the inclusion of the spatial lag term to the model might decrease the estimated parameter of 
the $\beta$-convergence. Such change is interpreted as the indirect influence of the trade relations, factor mobility and knowledge spillover in the convergence. In our models we do not observe any difference in the estimated parameters $\beta_{1}$ and $\beta_{2}$ after taking into account the spatial dependence. While in models 1 and 2 such a result is obvious as the spatial interaction parameters are insignificant, no change of the $\beta$-convergence parameters in model 3 might suggest that the conditional $\beta$-convergence that we observe in the metros and non-metros was not supported by the existence of the spatial interactions among subregions in the period 2000-2010.

\subsection{Two-regime spatial autoregressive panel models}

Finally, we estimated models with the two-regime spatial dependence and four different combinations of the spatial and time fixed effects. The estimation results are presented in Table 3 . Let us now concentrate only on the results from models $1-3$.

Table 3

Estimation results for conditional $\beta$-convergence and spatial regimes using two-regime spatial autoregressive panel models.

\begin{tabular}{|c|c|c|c|c|}
\hline Parameter & Model 1 & Model 2 & Model 3 & Model 4 \\
\hline$\beta_{1}$ & $\begin{array}{l}-0.023 \\
(0.002)\end{array}$ & $\begin{array}{c}-0.017 \\
(0.004)\end{array}$ & $\begin{array}{c}-0.026 \\
(0.003)\end{array}$ & $\begin{array}{l}-0.014 \\
(0.003) \\
\end{array}$ \\
\hline$\beta_{2}$ & $\begin{array}{l}-0.025 \\
(0.002)\end{array}$ & $\begin{array}{c}-0.030 \\
(0.003)\end{array}$ & $\begin{array}{c}-0.021 \\
(0.002)\end{array}$ & $\begin{array}{l}-0.032 \\
(0.003)\end{array}$ \\
\hline$\rho_{1}$ & $\begin{array}{c}0.402 \\
(0.200)\end{array}$ & $\begin{array}{c}0.272 \\
(0.234)\end{array}$ & $\begin{array}{c}0.886 \\
(0.032)\end{array}$ & $\begin{array}{c}0.407 \\
(0.183)\end{array}$ \\
\hline$\rho_{2}$ & $\begin{array}{c}0.310 \\
(0.140)\end{array}$ & $\begin{array}{c}0.281 \\
(0.146)\end{array}$ & $\begin{array}{c}0.901 \\
(0.019)\end{array}$ & $\begin{array}{c}0.532 \\
(0.108)\end{array}$ \\
\hline Spatial FE & Yes (1) & Yes (2) & No & Yes (3) \\
\hline Time FE & Yes (1) & Yes (2) & Yes (2) & Yes (2) \\
\hline Metros dummy & No & No & No & Yes \\
\hline$\sigma_{\mu}^{2}$ & $\begin{array}{l}0.0001 \\
(0.000)\end{array}$ & $\begin{array}{c}0.0001 \\
(0.000)\end{array}$ & $\begin{array}{c}0.0001 \\
(0.000)\end{array}$ & $\begin{array}{l}0.0001 \\
(0.000)\end{array}$ \\
\hline$\sigma_{\varepsilon}^{2}$ & $\begin{array}{c}0.003 \\
(0.000) \\
\end{array}$ & $\begin{array}{c}0.003 \\
(0.000) \\
\end{array}$ & $\begin{array}{c}0.003 \\
(0.003) \\
\end{array}$ & $\begin{array}{c}0.003 \\
(0.000) \\
\end{array}$ \\
\hline \multicolumn{5}{|l|}{ Metro: } \\
\hline conv. speed & $2.327 \%$ & $1.715 \%$ & $2.634 \%$ & $1.410 \%$ \\
\hline half-time & 29.789 & 40.426 & 26.311 & 49.163 \\
\hline \multicolumn{5}{|l|}{ Non-metro: } \\
\hline conv. speed & $2.532 \%$ & $3.046 \%$ & $2.122 \%$ & $3.252 \%$ \\
\hline half-time & 27.378 & 22.757 & 32.659 & 21.312 \\
\hline
\end{tabular}

Posterior means, posterior standard deviations in parentheses. (1) one-regime effects, (2) tworegime effects,(3) only for the non-metros

Source: author's calculations. 
Incorporating the two-regime spatial dependence does not affect the estimated random effect variance as well as the error term variance. Also the estimated $\beta$ coefficients in models 1,2 and 3 are the same as in the previous models (compare with Table 2), which suggests no impact of the diversification of the spatial interactions parameter on the convergence process. Hence, the significant difference in the conditional $\beta$-convergence between metros and non-metros is found only when the two-regime spatial fixed effects are applied.

The separation of the spatial interaction parameter into: 1) connected with the interactions with metros, 2) representing the impact of neighbours on the non-metros, results in the significant diversification of the estimated coefficients value in the model with one-regime spatial fixed effects (denoted by 1 in Table 3). It seems that the insignificance of a parameter for the spatial dependence in the spatial random effect model (denoted by 1 in Table 2) is connected with the lack of the spatial interactions in the metros subsample. For the non-metros the spatial interactions exist because the $\rho_{2}$ parameter is significant.

Despite this, a significant difference between the value of the estimated $\rho_{1}$ and $\rho_{2}$ coefficients was not found when the two-regime spatial fixed effects are applied. In such model (denoted by 2 in Table 3 ) both for the metros and nonmetros there are no spatial interactions. This result is consistent with those from the model with one-regime spatial dependence (denoted by 2 in Table 2). The results from the models without the spatial fixed effects are also the same. When we assumed there are no country-specific effects, the one-regime as well as the two-regime spatial interaction parameters are significant. In the model with tworegime spatial dependence both $c_{1}$ and $\rho_{2}$ parameters are significant but there is no significant difference between their values.

Again, we can observe that the significance of the spatial interaction parameters depends on how we applied the country-specific effects. The form in which the spatial fixed effects occur in the models affects also the estimated parameters of the $\beta$-convergence. The diversification of the country-specific effects into one or two spatial regimes seems to play a crucial role. When the country effects are separated for the metros and nonmetros, the parameters for the conditional $\beta$-convergence vary but the parameters for the spatial interactions are equal and insignificant. In contrast, when we allow for the common country-specific effects there is no significant difference in the conditional $\beta$-convergence between metros and non-metros, but the parameters for the spatial interactions are unequal and significant for the non-metros $\left(\rho_{2}\right)$. 
Due to the above, we estimated an additional model with the countryspecific effects for non-metros and additional dummy variable with the value 1 if the subregion is the metro and 0 otherwise. Hence, we captured the difference between metros and non-metros in the economic growth, as well as allowed for its variation among the non-metros localized in different countries. In contrast to the models with the two-regime spatial fixed effects, we assumed the effect of being localized in the same country does not occur for the metros. Despite this, as long as the random effects are in the model, we allowed for the individual treatment of each subregion, for example in the initial level of technology. This model is denoted by 4 in Table 3 .

The results suggest the estimated parameter for the spatial interactions is significant for the non-metros and insignificant for the metros. Also we found the estimated parameter for the conditional $\beta$-convergence is significantly higher for the metros $\left(\beta_{1}=-0.014\right)$ than for the non-metros $\left(\beta_{2}=-0.032\right)$. The $\beta$ coefficient estimates are equal to those achieved by using the model with two-regime spatial fixed effects, while the values of the $\rho$ coefficient estimates are the same as in the model with one-regime spatial fixed effects. Hence we can argue that in model 4 we obtain such a structure of the spatial fixed effects which has some features of the one and tworegime approaches. Finally, it seems that the assumptions about the differences between metros and non-metros as well as between countries in the specific effects play a crucial role in the verification of our hypothesis.

\section{DISCUSSION}

This section provides additional discussion about the heterogeneity of the convergence speed and spatial dependence expressed by the specific spatial effects. Moreover, we would like to compare our results with those obtained in similar studies by other authors.

\subsection{Heterogeneity of the convergence process}

According to the last model (Table 3) the estimated speed of the convergence to the individual steady state was found to be lower for the metros (1.4\%) and higher for the non-metros (3.3\%). Our results are within the range found by Barro and Sala-i-Martin (1995). As the number of metros and their importance differs strongly between the EU-15 and newcomers, some of the basic comparisons with the convergence process among old and new members of the EU might be done. Most similar within the results are 
Chapman et al. (2012), who achieved a convergence speed of over $2 \%$ for the newcomers and only $1.59 \%$ for the EU-15 regions during 1995-2004.

In all of the models the estimates for the conditional $\beta$-convergence parameters seem to be unaffected by the omitted spatial dependence. However, we found a strong relationship between the $\beta_{1}$ and $\beta_{2}$ parameters and the country specific effects. This suggests that when the national factors are included, the catch-up of some subregions might slow down or speed up. Similarly to us, Paas et al. (2007) showed that taking countries' structural characteristics into account might change the speed of the convergence, which means the catching up process is driven mostly by country-specific factors.

An additional conclusion from our research is that the change in the catching up process after controlling for the national effects is different for the metros and non-metros. When we allow for the two-regime countryspecific effects, the speed of the convergence for the metros decreased significantly, while for the non-metros we noticed its significant increase (model 2, Table 3). The same results were achieved when the countryspecific characteristics for the metros were removed (model 4, Table 3). The convergence of metros seems to be more independent from the countryspecific factors. This is because there is no significant difference between models 2 and 4 in the speed of the convergence for the metros. In contrast, the country-specific effects seem to be the powerful modifiers for the catching up process among the non-metros. When we allowed for the national effects, the speed of the convergence for the non-metros increased from $2.1 \%$ to $3.3 \%$ (compare the results from model 3 and 4 ).

\subsection{Difference in spatial dependence between metros and non-metros}

For model 4 with the metro dummy variable and the national effects for the non-metros, as well as for model 1 with the one-regime spatial fixed effects, we found a significant difference in the parameter of spatial interaction. In both models the significant spatial interactions parameter was proved only for the non-metros. This proves the heterogeneity of spatial dependence between metros and non-metros, however we achieved it only for the two particular specification of the spatial fixed effects. The differentiation of the spatial dependence into regimes seems to be unnecessary due to the results from models 2 and 3.

In model 2, spatial autocorrelation of the non-metro units seems to be sufficiently captured by the country dummies because the estimated $\rho_{2}$ 
parameter is insignificant. Lack of significance for the spatial interaction parameter after allowing for the unidentified national macroeconomic factors was found also by Paas et al. (2007). Like them, we noticed the countryspecific effects are more important for subregional convergence than spatial interaction. An additional argument for this conclusion is that there is no significant change of the $\beta$-convergence parameters after incorporating the spatial lag term.

Our results differ from Baumont et al. (2006), who proved the existence of the spatial regimes as the club-convergence but denied the need of differentiating spatial effects for the North and South European regimes (separated by using the NUTS2 level data for the period 1980-1995). Still we are quite consistent with Mur et al. (2010), who proved the instability (as the Centre-Periphery dichotomy) of the parameters of $\beta$-convergence as well as parameters of spatial dependence.

Although spatial interactions for the metros were not proved, it is hard to expect no spillover of knowledge and impact of trade links or factors mobility for those units. The insignificance of the $\rho_{1}$ parameter might be the result of the low cut-off distance in the $\mathbf{W}$ matrix specification. While the semi-local structure of spatial interactions for the non-metros might be enough to capture relationships with those units, it is possible that for the metros the geographical distance plays a minor role and the global structure of spatial interactions would be more appropriate.

\section{CONCLUSIONS}

In this paper we focused our attention on the heterogeneity of the $\beta$-convergence process and the instability of spatial dependence between metropolitan and non-metropolitan areas of Europe. We proposed the tworegime spatial autoregressive panel data model estimated in the Bayesian framework to examine the difference between metros and non-metros. A small Monte Carlo study was conducted to evaluate the unbiasedness of its parameters. Our research was based on a sample of 1,363 European subregions (for the period 2000-2010), classified as metro or non-metro according to the LUZ delimitation.

The findings suggest a significant catching up process for both metros and non-metros during 2000-2010. After controlling the country-specific effects for non-metros and separating the metro/non-metro by using the dummy variable, we observe a significant difference between metropolitan and non-metropolitan areas in the speed of the convergence. According to 
our results the half-time rate is 49 years for the metros and 21 for the nonmetros. Additionally, we noticed the predominant role of the countryspecific characteristics (e.g. technology, political environment) for the conditional $\beta$-convergence, especially among the non-metros.

The effect of spillover was found only for the non-metros, while for the metros the spatial interaction parameter was insignificant. Again, the different way of specifying the spatial fixed effects results in the difference between the estimated $\rho_{1}$ and $\rho_{2}$ parameter. For those models in which spatial interactions parameters were found as insignificant, we can conclude that the existing spatial autocorrelation has been captured by the country dummies. Hence we need to be careful when drawing more general conclusions about the instability of spatial dependence between metros and non-metros. Finally, we did not find any proof suggesting that the spatial spillover supports the convergence process.

\section{REFERENCES}

Akhmedjonov, A., Lau, M. C. K., İzgi, B.B., New Evidence of Regional Income Divergence in Post-reform Russia, "Applied Economics", 45(18), pp. 2675-2682, 2013.

Anselin, L., Spatial Econometrics: Methods and Models. Studies in Operational Regional Science. Kluwer Academic Publishers, Netherlands, 1988.

Arbia, G., Basile, R., Piras, G., Using Spatial Panel Data in Modelling Regional Growth and Convergence, Istituto di Studie Analisi Economica, Working paper, Vol 5, pp. 8-31, 2005.

Badinger, H., Müller, W. G., Tondl, G., Regional Governance in the European Union (1985-1999): A Spatial Dynamic Panel Analysis, HWWA Discussion Paper, Vol. 210, pp. 1-252, 2002.

Barro, R., Sala-i-Martin, X., Convergence across States and Regions, "Brookings Papers on Economic Activity", pp. 107-182, 1992.

Barro R., Sala-I-Martin, X., Economic Growth. McGraw Hill, New York, 1995.

Baumont, C., Ertur, C., Le Gallo, J., The European Regional Convergence Process, 1980-1995: Do Spatial Regimes and Spatial Dependence Matter?, "International Regional Science Review", 29.1, pp. 3-34, 2006.

Bolstad, W. M., Understanding Computational Bayesian Statistics (Vol. 644). John Wiley \& Sons, 2011.

Bouayad-Agha, S., Védrin, L., Estimation Strategies for a Spatial Dynamic Panel Using GMM. A New Approach to the Convergence Issue of European Regions, "Spatial Economic Analysis", 5(2), pp. 205-227, 2010.

Caselli, F., Esquivel, G., Lefort, F., Reopening the Convergence Debate: A New Look at Cross-country Growth Empirics, "Journal of Economic Growth", 1, pp. 363-389, 1996.

Chapman, S. A., Cosci, S., Mirra, L., Income Dynamics in an Enlarged Europe: The Role of Capital Regions, "The Annals of Regional Science", 48(3), 2012. 
Dańska-Borsiak, B., Konwergencja czy dywergencja polskich województw? [Convergence or divergence of Polish voivodeship?], „Acta Universitatis Lodziensis. Folia Oeconomica”, 253, pp. 41-54, 2011.

Dijkstra, L., Metropolitan Regions in EU, "Regional Focus", European Commission, Regional Policy, 2009.

Dong, G., Harris, R, J., Spatial Autoregressive Models for Geographically Hierarchical Data Structures, "Geographical Analysis", 2014.

Elhorst, J. P., Fréret, S., Evidence of Political Yardstick Competition in France using a Tworegime Spatial Durbin Model with Fixed Effects, "Journal of Regional Science", 49(5), pp. 931-951, 2009.

Eurostat, Eurostat regional yearbook 2013, available at: http://epp.eurostat.ec.europa.eu/ cache/ITY_OFFPUB/KS-HA-13-001/EN/KS-HA-13-001-EN.PDF, 2013.

Fischer, M. M., Stirböc, C., Pan-European Regional Income Growth and Club-convergence, "The Annals of Regional Science", 40(4), pp. 693-721, 2006.

Frenken, K., Hoekman, J., Convergence in an Enlarged Europe: The Role of Network Cities, "Tijdschrift voor economische en sociale geografie", 97(3), pp. 321-326, 2006.

Geodecki, T., Procesy konwergencji $i$ polaryzacji $w$ regionach Unii Europejskiej [The proocesses of convergence and polarization in EU regions], Zeszyty Naukowe Akademii Ekonomicznej w Krakowie, nr 714, Kraków, 2006.

Hammond, G. W., A Time Series Analysis of US Metropolitan and Non-metropolitan Income Divergence, "The Annals of Regional Science", 40(1), pp. 81-94, 2006.

Hays, J. C., Kachi, A., Franzese, Jr. R. J., A Spatial Model Incorporating Dynamic, Endogenous Network Interdependence: A Political Science Application, "Statistical Methodology", 7(3), pp. 406-428, 2010.

Hoogland, J., Boomsma, A., Robustness Studies in Covariance Structure Modeling: An 14 Overview and a Meta-analysis, "Sociological Methods and Research", 26(3), 1998.

Jabłoński, Ł., Kapitał ludzki a konwergencja gospodarcza [Human capital and convergence process]. Wydawnictwo CH Beck, 2012.

LeSage, J., Pace, R. K., Introduction to Spatial Econometrics. CRC press, 2010.

Mur, J., López, F., Angulo, A., Instability in Spatial Error Models: An Application to the Hypothesis of Convergence in the European Case, "Journal of Geographical Systems", 12(3), pp. 259$-280,2010$.

Nielsen, R., Statistical Methods in Molecular Evolution. Springer Science \& Business Media, 2006.

Paas, T., Kuusk, A., Schlitte, F., Econometrics Analysis of Income Convergence in Selected EU Countries and Their NUTS 3 Level Regions. University of Tartu, Faculty of Economics and Business Administration, Andres Võrk Tartu University Press, Tartu, 2007.

Pietrzak, M. B., Wykorzystanie przestrzennego modelu regresji przełacznikowej $w$ analizie regionalnej konwergencji w Polsce [An application of spatially switching regression model in an analysis of regional convergence in Poland], „Ekonomia i Prawo”, 11(4), pp. 167$-186,2012$.

Plummer, M., Best, N., Cowles, K., Vines, K., CODA: Convergence Diagnosis and Output Analysis for MCMC, "R news", 6(1), pp. 7-11, 2006. 
Pukeliene, V., Butkus, M., Evaluation of Regional $\beta$ Convergence in EU Countries at NUTS3 Level, "Ekonomika/Economics", 91(2), 2012.

Viegas, M., Antunes, M., Convergence in the Spanish and Portuguese NUTS 3 Regions: An Exploratory Spatial Approach, "Intereconomics", 48(1), pp. 59-66, 2013.

Williamson, J. G., Regional Inequality and the Process of National Development: A Description of the Patterns, "Economic Development and Cultural Change", 13 (2), 1965.

Yu, J., Lee, L. F., Convergence: A Spatial Dynamic Panel Data Approach, "Global Journal of Economics", 1(01), 2012.

Received: September 2014

Acknowledgements: This work was financially supported by grants ETIUDA1 from the Polish National Centre for Science, UMO-2013/08/T/HS4/00512. 